\title{
Théâtre, tragédie et raison pure. Sur le $\$ 52$ de la Critique de la faculté de juger de Kant
}

\section{Olivier Dubouclez}

\section{(2) OpenEdition}

1 Journals

Édition électronique

URL : https://journals.openedition.org/edl/3163

DOI : $10.4000 /$ edl. 3163

ISSN : 2296-5084

\section{Éditeur}

Université de Lausanne

\section{Édition imprimée}

Date de publication : 24 septembre 2020

Pagination : 79-96

ISBN : 978-2-940331-74-1

ISSN : 0014-2026

\section{Référence électronique}

Olivier Dubouclez, «Théâtre, tragédie et raison pure. Sur le §52 de la Critique de la faculté de juger de Kant », Études de lettres [En ligne], 313 | 2020, mis en ligne le 24 septembre 2021, consulté le 25 septembre 2021. URL : http://journals.openedition.org/edl/3163; DOI : https://doi.org/10.4000/edl. 3163 


\section{THÉÂTRE, TRAGÉDIE ET RAISON PURE. SUR LE $\$ 52$ DE LA CRITIQUE DE LA FACULTÉ DE JUGER DE KANT}

Kant traite du théâtre à la fin de la première partie de la Critique de la faculté de juger, au $\$ 52$ qui est consacré à «l'association des beaux-arts». Il y met en lumière une double précarité: celle du théâtre conçu comme éloquence (ce rapprochement le tirant du côté du simple divertissement), et celle de la tragédie, genre sublime, constamment menacée d'être réduite à une exaltation des forces vitales. Kant réfléchit alors sur les conditions historiques du surgissement du sentiment tragique: sa sublimité dépend en effet d'une double condition, subjective (du côté du spectateur comme sujet moral), et de l'artiste (en tant qu'il est capable de présenter l'infini) au sein d'un devenir culturel où s'accomplit la moralisation de l'être humain.

À l'évidence, Kant parle peu du théâtre dans la Critique de la faculté de juger (1790). Ce qui n'est pas la conséquence d'un oubli ni d'une méconnaissance, mais plutôt d'une décision théorique: vers 1776-1778, Kant voyait dans le théâtre un art élémentaire et le situait "parmi les arts qui relèvent du goût" ${ }^{1}$; plus tôt encore, en 1764 , ses Observations sur le sentiment du beau et du sublime traitaient de la tragédie comme d'une forme artistique sublime et faisaient référence aux situations morales dépeintes par celle-ci ${ }^{2}$. Avec la Critique de la faculté de juger, la rupture est nette: le théâtre n’est plus un art (il n’est pas répertorié au $\$ 51$ parmi les beaux-

I. D. Dumouchel, Kant et la genèse de la subjectivité esthétique, p. 245. Cf. I. Kant, Reflexionen zur Anthropologie, R983, in Kant's gesammelte Schriften, vol. 15-1, p. 429.

2. "Dans la première [la tragédie] on voit le sacrifice magnanime en faveur d'autrui, l'esprit de décision hardi dans le danger et une fidélité éprouvée» (E. Kant, Observations sur le sentiment du beau et du sublime, in E. Kant, Euvres philosophiques, vol. 1, p. 457). 
arts) ${ }^{3}$ et la tragédie, comme on va le voir, fait l'objet d'un traitement négatif et déceptif qui rompt avec sa première conception.

Pour le comprendre, il faut rappeler de quoi parle la troisième Critique: non pas d'œuvres d'art particulières (Kant n'en cite quasiment aucune), mais d'objets, c'est-à-dire de dispositifs empiriques offerts à notre perception dont l'unité est assurée par une forme, et qui sont l'occasion d'un plaisir de nature particulière, le plaisir esthétique. C'est de cette façon que le théâtre est abordé dans le $\$ 52$ : il n'y a pas d'art théâtral, mais il y a des pièces de théâtre qui sont autant d'exemples de «l'association (Verbindung) des beaux-arts en un seul et même produit " ${ }^{4}$; c'est aussi le cas du chant, de la danse ou de l'opéra qui ne sont pas des arts primaires, mais des ensembles d'objets composites et synesthésiques (puisqu'ils s'adressent en même temps à plusieurs sens). Kant pense donc le théâtral du point de vue de ce qu'on appellerait aujourd'hui son «intermédialité», au seuil de la grande tradition du Gesamtkunstwerk qui trouvera ses racines dans l'esthétique de Schiller ${ }^{5}$.

Une fois reconnue cette situation particulière, on peut tenter de lire le $\$ 52$, situé à l'extrême pointe de l'«Analytique de la faculté de juger esthétique», comme le lieu d'une détermination précise de la valeur esthétique et morale du théâtre et, plus particulièrement, de sa forme tragique. Cette détermination exigera que l'on recompose le paysage théorique dans lequel s'inscrit ledit paragraphe et que l'on explicite les idées et les thèses qui s'y trouvent présupposées.

\section{Précarité des ouvres composites}

Précisons d'emblée la perspective sous laquelle se pose pour Kant la question du théâtre: celle de l'unité de l'œuvre, qu'on l'entende en un sens formel (qu'est-ce qui fait l'unité de l'objet produit?) ou en un sens affectif (comment ses multiples effets contribuent-ils à une expérience sensible unifiée?). Le $\$ 52$ distingue en effet deux types d'«association » : celle des arts dans la composition des œuvres et celle des sentiments dans leur

3. E. Kant, Critique de la faculté de juger, \$51, p. 307-312.

4. Ibid., p. 313. Toutes les références au $\$ 52$ renverront à cette même page. Pour le texte allemand, voir Kant's gesammelte Schriften, vol. 5, p. 325 sq.

5. Voir M. Groneberg, "Le Gesamtkunstwerk et l'homme entier». 
appréciation. S'agissant du premier type, Kant fournit trois exemples qui chacun mettent en jeu les trois grandes catégories kantiennes des beauxarts: "art de la parole», "art figuratif» et "art du jeu des sensations» (auquel se rapporte notamment la musique) ${ }^{6}$. Le théâtre combine art de la parole et art figuratif, le chant art de la parole et art du jeu des sensations et la danse art du jeu des sensations et art figuratif ${ }^{7}$. Kant indique aussi la possibilité d'une combinatoire au second degré: l'opéra comme théâtre chanté ${ }^{8}$. À côté de cette combinaison des arts, il distingue une association des sentiments liés aux catégories esthétiques du beau (das Schöne) et du sublime (das Erhabene) qu'il a appris à distinguer à la suite des travaux de Burke. Pour Kant, le plaisir esthétique relève d'une expérience qui met en jeu les facultés du sujet, dans leur harmonie spontanée (c'est le cas du plaisir ressenti devant le beau) ou au contraire dans une dysharmonie qui, liée au débordement de l'imagination, nourrit alors le "plaisir négatif" du sublime 9 . Dans les deux cas, la satisfaction éprouvée est universalisable et donc attribuable à autrui. À l'inverse, l'agréable, c'est-à-dire le plaisir lié à la stimulation des sens relève de «l'attrait» (Reiz) et cause une jouissance de rang inférieur, strictement individuelle. Signalons un autre point: les sentiments du beau et du sublime mobilisent des facultés différentes et trouvent leur origine dans des circonstances empiriques distinctes, voire incompatibles puisque l'un implique la perception d'une forme déterminée (ainsi la belle fleur) et l'autre une expérience de l'informe et de l'illimité (ainsi l'océan déchaîné) ${ }^{10}$. La surprise est donc grande de voir que le $\$ 52$ affirme la possibilité de leur union:

[...] la présentation du sublime (die Darstellung des Erhabenen), dans la mesure où elle appartient aux beaux-arts, peut s'unir (verbunden werden) avec la beauté (Schönheit) dans une tragédie en vers (im gereimten Trauerspiele), un poème didactique, un oratorio.

6. E. Kant, Critique de la faculté de juger, \$51, p. 307.

7. Ibid., $\$ 52$, p. 313. Selon le $\$ 14$, la danse combine le jeu des sensations musicales et le «jeu des figures» (p. 204) que Kant a rapporté à la peinture, comme c'est aussi le cas de la «mimique» (Ibid.).

8. Sur cette combinatoire et l'ensemble du $\$ 52$, on peut aussi se reporter à la lecture puissante de J.-Y. Chateau (Pourquoi un septième Art?, p. 48-73), ainsi qu'aux analyses d'E. Kirkkopelto (Le théâtre de l'expérience, p. 103-147).

9. Voir Critique de la faculté de juger, \$23, p. 226.

Io. Ibid., p. 225 sq. 
Affirmation immédiatement tempérée par un doute qui va orienter l'ensemble de la réflexion:

[...] et dans ces associations les beaux-arts sont encore plus artistiques (künstlicher): quant à savoir toutefois s'ils sont aussi plus beaux (dans la mesure où viennent s'y entrecroiser maintes sortes de satisfactions si diversifiées), cela peut demeurer douteux dans certains cas.

L'association des arts crée, dans un art synthétique, un surplus d'artificialité $^{11}$. En effet, s'il y a plus d'artificialité dans le chant que dans la poésie ou dans la musique prises séparément, c'est parce qu'à la contrainte d'unir une forme artistique à une matière s'ajoute alors la contrainte de synthétiser par une forme englobante, celle du chant, les formes musicale et poétique. Cet effet cumulatif se retrouve du côté des «satisfactions»: le chant nous donne le plaisir de la musique, mais aussi celui de la poésie, plaisirs qui, du reste, ne sont pas nécessairement du même ordre (la musique peut relever du beau, mais aussi de l'agréable). La satisfaction ne se simplifie pas: c'est même ce qui fait la richesse de cette expérience esthétique, que l'on jouisse du "produit» sous différents aspects. Or le doute kantien porte justement sur cet effet d'accumulation: est-ce que les satisfactions esthétiques, en se cumulant, accroissent la satisfaction prise à la considération du tout? Est-ce qu'une ouvre belle et présentant le sublime ${ }^{12}$, c'est-à-dire où "une dimension d'illimité est représentée en [elle] ou grâce à [elle] " ${ }^{13}$, est plus belle qu'une ouvre simplement belle? Si Kant pose cette question, c'est pour une raison de fond: "Dans tous les beaux-arts, l'essentiel réside dans la forme», rappelle le $\$ 52$, puisque la forme est ce qui assure son unité ${ }^{14}$. Donc le risque d'avoir une ouvre à la fois picturale et poétique, ou bien belle et en même temps sublime, c'est qu'elle soit plus riche, mais aussi plus composite, moins unifiée, et donc moins satisfaisante en tant que tout. Le doute kantien nous place devant une ambiguïté: les œuvres combinées peuvent résulter chacune

II. Künstlich se dit d'un objet produit par rapport à des règles: "Car tout art (Kunst) suppose des règles par le truchement desquelles seulement un produit est représenté comme possible, s'il doit être désigné comme un produit de l'art (wenn es künstlich heissen soll)» (Critique de la faculté de juger, $\$ 46$, p. 293).

I2. Nous reviendrons sur cette expression dans notre point 3.

13. Critique de la faculté de juger, \$23, p. 225.

I4. Voir à nouveau le $\$ 14$ où le dessin est en effet «l'essentiel (das Wesentliche)» (Ibid., p. 204). 
en une belle totalité, mais cet aboutissement est particulièrement précaire du fait qu'il n'existe pas de forme a priori du théâtre, de la danse, de l'opéra, etc. Le fait que, hormis le poème didactique, Kant se réfère à des œuvres impliquant un événement scénique (tragédie en vers et oratorio) ajoute à ce sentiment que le propre de l'art fondé sur une Verbindung, en raison même de son hétérogénéité, c'est qu'il puisse manquer son effet.

\section{La pièce de théatre et le divertissement}

Il est intéressant de noter qu'à côté de cette précarité liée au caractère composite de l'œuvre intermédiale se trouve une autre précarité, présente dans la définition de la pièce de théâtre. Celle-ci consiste selon Kant en l'association de "l'éloquence» à "une présentation picturale (malerische Darstellung) de ses sujets (seiner Subjekte) aussi bien que de ses objets (seiner Gegenstände)». Pour comprendre la notion de "présentation picturale», il faut rappeler que la présentation est «le fait de placer à côté du concept une intuition correspondante", ce qui peut s'effectuer, précise l'introduction de la troisième Critique, "par l'intermédiaire de notre propre imagination, comme dans l'art, quand nous réalisons un concept formé au préalable d'un objet qui constitue pour nous une fin ${ }^{15}$. Au théâtre, la "présentation picturale» consistera en la représentation par le moyen de la peinture d'un concept mentionné dans le texte dramatique, sous la forme d'un élément visible sur la scène. Il faut mettre en rapport cette notion de "présentation picturale» avec le sens élargi de la peinture envisagé dans le $\$ 51$ qui relève alors du «bel agencement (Zusammenstellung)" des "produits" de la nature ou "bel agencement des choses physiques» ${ }^{16}$. La "présentation picturale» désigne donc un élément formel, et non matériel, de la représentation: un agencement esthétique d'«objets» et de personnes en habits (les «sujets») qui prendra la forme d'un «tableau» commandé par les concepts propres à la pièce de théâtre considérée.

15. Ibid., p. 172. Voir aussi la «Première introduction» (Ibid., p. 110).

I6. "Au compte de la peinture au sens large, je mettrais encore la décoration des pièces par des tapisseries, des garnitures, et tout bel ameublement qui est destiné simplement à la vue; de même l'art de s'habiller avec goût (bagues, tabatières, etc.) " de manière, précise Kant, à agencer "une sorte de tableau» (Ibid., p. 310). 
C'est toutefois dans le premier point de sa définition, son rapport à «l'éloquence» que le théâtre se montre à nouveau précaire. Selon un rapprochement habituel, mais qui est justement remis en cause au cours du XVIII ${ }^{e}$ siècle, Kant identifie l'acteur et l'orateur ${ }^{17}$. À la différence du théâtre, l'éloquence est pour Kant un art élémentaire qu'il oppose diamétralement à cet autre art de la parole qu'est la poésie ${ }^{18}$. Le $\$ 51$ définit l'éloquence comme «l'art de mener à bien une opération de l'entendement comme s'il s'agissait d'un libre jeu (Spiel) de l'imagination " par opposition à la poésie qui est «l'art de mener à bien un libre jeu de l'imagination comme s'il s'agissait d'une opération de l'entendement»" ${ }^{19}$. L'orateur, avec son discours en prose, argumente et fait réfléchir, mais dans une forme séduisante, de sorte que le but sérieux de son activité, argumenter, est dégradé par la forme divertissante qu'il lui donne. Il parle d'un sujet sérieux, mais il le fait en badinant. À l'inverse, le poète qui annonce un "jeu divertissant (ein unterhaltendes Spiel)» se met à produire des images qui finissent par nous "faire penser", comme le rend possible la mobilisation de ce que Kant appelle «l'Idée esthétique» ${ }^{20}$. L'éloquence manque donc à sa "promesse" ${ }^{21}$ : elle contient en elle le mouvement d'une dégradation puisqu'au lieu de «mettre en œuvre l'entendement d'une façon qui soit conforme à une fin » ${ }^{22}$ et de nous amener à l'élargissement du "faire penser", elle s'en tient à un simple jeu. Le théâtre, qui relève de l'éloquence, ne se trouve-t-il pas lui aussi dévalorisé? On peut le craindre, mais la "tragédie en vers" fait manifestement exception. En précisant que la tragédie est versifiée, Kant la fait glisser du côté de la poésie et donc résout le risque de dégradation propre à sa dimension oratoire. L'importance du vers dans ce contexte sera soulignée par l'Anthropologie:

17. Sur l'histoire des rapports entre orateur et acteur au XVIII e siècle, voir la synthèse de L. Marie, Inventer l'acteur, ainsi que Ch. de Gemeaux, De Kant à Adam Müller (1790-1815).

I8. Le $\$ 49$ ne les dissociait pas encore, mais les associait au titre de ce qui fait "penser davantage" que le simple concept (Critique de la faculté de juger, p. 302).

I9. Ibid., p. 308.

20. Ibid., $\$ 49$, p. 301; $\$ 53$, p. 314 . Sur la différence importante entre Unterhaltung et Zerstreuung, voir J.-Y. Chateau, Pourquoi un septième art?, p. 50.

2I. E. Kant, Critique de la faculté de juger, p. 308.

22. Ibid. 
Pourquoi entend-on d'ordinaire par poète celui qui compose des vers, c'est-à-dire un discours scandé (parlé sur un mode musical et rythmé)? Parce qu'annonçant une œuvre d'art, il se présente avec une solennité (Feierlichkeit) qui doit suffire au goût le plus fin (pour la forme); autrement, il n'y aurait point de beauté. Mais comme cette solennité est exigée surtout pour la belle représentation du sublime, une telle solennité, si elle est affectée et n'utilise pas le vers, est désignée (par Hugh Blair) comme de la "parole en délire» ${ }^{23}$.

Le vers fait donc deux choses: il prévient la chute dans le divertissement qui caractérise l'éloquence et, ce faisant, il pose le cadre propice aux emportements du discours sublime. Versification qui se retrouve en effet dans les formes citées par Kant (tragédie, oratorio, poème didactique) qui, toutes, savent atteindre au sublime.

\section{La tragédie, entre sublime et déception}

Pareille atteinte du sublime doit cependant être interrogée. Elle suscite doublement l'étonnement dans un cadre kantien ${ }^{24}$. D'abord parce que Kant pose comme un fait et comme un fait pluriel l'existence d'une œuvre d'art sublime, et même, on l'a vu, belle et sublime. Or le sublime se rapporte rarement à l'art dans la Critique de la faculté de juger: Lorsque Kant parle du sublime, il le fait la plupart du temps en rapport avec le sublime de la nature, à l'exception de quelques renvois au sublime architectural ${ }^{25}$. Il existe un seul passage où il est question du sublime dans les arts littéraires, dans le $\$ 49$ (qui précède de quelques pages le texte que nous étudions): lorsque le poète forge un vers suscitant la «conscience de la vertu», il "répand dans l'esprit une foule de sentiments sublimes" et ouvre "sur un avenir de joie, tel qu'aucune expression adéquate à un concept déterminé ne l'atteint pleinement ${ }^{26}$. Situation paradoxale - l'expression ouvrant sur l'inexprimable - qui est en rapport

23. E. Kant, Anthropologie d'un point de vue pragmatiqu, p. 106.

24. Plus généralement, sur les rapports entre sublime et tragique, voir B. de Saint Girons, «Du sublime comme assomption du tragique».

25. Voir en particulier $\$ 26$, p. 233 sq.

26. E. Kant, Critique de la faculté de juger, \$49, p. 302 sq. 
direct, comme nous le verrons, avec la "présentation du sublime» dont parle le $\$ 52$.

Le second motif d'étonnement est que la présence du sublime dans la tragédie a beau être affirmée par Kant, son association avec le beau, on l'a vu, demeure néanmoins précaire. Le $\$ 52$ est curieusement hésitant, comme si la "tragédie en vers", capable d'atteindre au sublime, s'exposait pourtant à une perception incomplète ou inadéquate de sa complexité. Cette ambivalence se rencontrait déjà dans la «Remarque générale sur l’exposé des jugements esthétiques réfléchissants" qui suit le $\$ 29$, Kant convoquant la tragédie au cœur de sa réflexion sur le sublime, mais pour dire précisément qu'elle échoue à le susciter ${ }^{27}$. Comment comprendre ce geste: que la tragédie participe du sublime et que Kant doute pourtant qu'elle puisse y accéder (\$52) et constate même son échec (\$29)?

Commençons par la "Remarque générale». Kant y considère ce qu'il appelle les "affects sublimes» et traite d'abord de l'enthousiasme qui, s'il n'est pas un "état d'esprit (Gemütszustand)" sublime, mais un débordement "aveugle» et irréfléchi, acquiert une tout autre valeur lorsqu'il est vu de l'extérieur. Le spectacle de l'enthousiasme chez autrui est sublime en tant que s'y montre une "tension des forces grâce à des Idées" qui donnent à l'esprit un "élan (Schwung)" plus fort que l'impulsion sensible (Antrieb), le rapprochant du sublime dynamique où se révèle, face au déchaînement de la nature, la force intime du spectateur qui découvre ainsi sa grandeur morale ${ }^{28}$. Kant élargit alors son enquête et oppose deux types d'affects. Le "genre courageux", écrit-il, "éveille en nous la conscience des forces qui nous permettent de vaincre toute résistance (Widerstand) ${ }^{29}$. Le spectacle du courage dynamise le sujet humain, il lui dévoile "la force de l'esprit» qui peut s'opposer à n'importe quel obstacle sensible. Au contraire, le "genre languissant» aura tendance à le lénifier et l'incitera à renoncer «à toute confiance en [son] pouvoir de résister». La «sensiblerie (Empfindelei)» coupe de l'exigence morale -

27. Ibid., p. 257. D’une façon générale, chaque fois que Kant parle du théâtre dans la Critique de la faculté de juger, la tonalité est celle de la déception. On sait pourtant qu'il était amateur de spectacles, la vie théâtrale à Königsberg étant particulièrement riche (M. Kuehn, Kant, p. 166), mais il semble aussi qu'il y ait eu une rupture dans le comportement de Kant vis-vis de celui-ci après 1764 (Ibid., p. 156).

28. E. Kant, Critique de la faculté de juger, p. 255 sq. Sur le sublime dynamique, voir le $\$ 28$ (Ibid., p. 244 sq.).

29. Ibid., p. 256. Voir aussi l'exemple du chef de guerre dans le $\$ 28$, p. 245. 
c'est l'effet caractéristique des romans comme d'un certain type de «spectacles larmoyants» ou de sermons religieux qui sont, au sens littéral, démoralisants et antithétiques avec l'état d'esprit sublime ${ }^{30}$.

Kant prévient toutefois une confusion, et c'est ici que la tragédie va être mentionnée. En effet, il arrive que des pièces de théâtre ou des sermons suscitent des «mouvements d'esprits tumultueux (stürmische Gemütsbewegungen)» contribuant à dynamiser le sujet dans ses facultés sensible et imaginative, au bénéfice de la vie et de la "santé»" ${ }^{31}$. Cette dynamisation n'est toutefois pas du tout de la même nature que celle qui a lieu sur le plan moral: un spectacle jubilatoire, pour reprendre un terme dont est friand le spectateur contemporain, n'élèvera pas au sublime; si dynamisation il y a, elle se fera sur un plan strictement émotionnel, relevant du «jeu des affects (Spiel der Affekte)» et qui ne saurait donc "prétendre à l'honneur d'une présentation sublime» ${ }^{32}$. C'est à cet usage strictement affectif que s'en tiennent maints spectateurs de tragédie:

Ainsi bien des gens se croient édifiés par un sermon qui pourtant n'édifie rien (nul système de bonnes maximes); ou ils se croient rendus meilleurs (gebessert) par une tragédie, alors qu'ils sont simplement contents (froh) d'avoir eu la chance de chasser leur ennui. Aussi le sublime doit-il toujours entretenir une relation avec la manière de penser, c'est-à-dire avec des maximes recommandant de procurer à l'élément intellectuel et aux Idées de la raison la domination sur la sensibilité 33 .

Kant ne condamne pas complètement cet «usage» de la tragédie. Il lui a même fait une place dans sa première conception du théâtre, le renvoyant au Spiel der Empfindungen ${ }^{34}$, et il le fera encore dans son Anthropologie en évoquant le «jeu des émotions opposées [qui] anime chez le spectateur le principe de la vie en le soumettant à un mouvement

30. Ibid., p. 256. Voir aussi la critique du roman dans les Propos de pédagogie (E. Kant, Euvres philosophiques, vol. 3, p. 1179).

31. E. Kant, Critique de la faculté de juger, p. 257.

32. Ibid.

33. Ibid.

34. «Das Spiel der Eindrücke ist Musik/Das Spiel der Empfindungen: Roman, Theater" (I. Kant, Reflexionen zur Anthropologie, in Kant's Gesammelte Schriften, 15-1, p. 267). 
intérieur» ${ }^{35}$. La troisième Critique considère quant à elle que cette conception relève d'une simple "exposition physiologique » ${ }^{36}$ et qu'il est d'autant plus important de la circonscrire qu'elle peut alimenter la confusion: qu'il s'agisse de la tragédie ou du sermon ${ }^{37}$, on prend en effet souvent la production d'un bien-être vital pour une authentique amélioration de soi. Il est possible que Kant ait ici en vue la catharsis comprise comme une purge passionnelle et un «jeu des affects» qui, ainsi déterminée, ne saurait avoir un effet véritablement moral, contribuant à la «satisfaction de la raison pure ${ }^{38}$. La catharsis se situerait plutôt au niveau de la physiologie, c'est-à-dire des forces vitales, et n'engagerait donc en rien les facultés supérieures que sont l'entendement et la raison qui entrent en jeu dans les expériences respectives du beau et du sublime. Pareille critique rapproche Kant de Rousseau ${ }^{39}$ et le sépare d'un Schiller qui pensera une catharsis morale, capable de nous faire prendre conscience de notre liberté lorsque la scène montre la puissance morale qui triomphe des forces de la nature ${ }^{40}$. Mais il faut aussi préciser que, sur ce point, Kant a manifestement évolué: alors que les Observations sur le beau et le sublime soutenaient une approche cathartique et émotionnelle où le spectateur de la tragédie pouvait, comme chez Burke, atteindre au sentiment moral par la médiation de la sympathie ${ }^{41}$, la Critique de la faculté de juger ne développe plus cette voie qui se situe hors de l'horizon transcendantal. Ce qui est aussi la raison pour laquelle Kant ne parle plus en 1790, comme c'était le cas en 1764, de situations tragiques, de scènes de

35. E. Kant, Anthropologie d'un point de vue pragmatique, \$60, p. 94.

36. Sur l'opposition avec l'« exposition transcendantale», voir la fin de la «Remarque générale» (E. Kant, Critique de la faculté de juger, p. 260-262) ainsi que le $\$ 54$ (p. 318 sq.).

37. Voir S. R. Stroud, Kant and the Promise of Rhetoric, p. 118 sq. et 180.

38. E. Kant, Critique de la faculté de juger, p. 256.

39. Voir J.-J. Rousseau, Lettre à D’Alembert, p. 23 sq.

40. Voir J.-O. Bégot, «La naissance du tragique, enfanté par l'esprit du sublime».

4I. Le passage des Observations cité plus haut (note 2) se poursuit en effet ainsi: «L'amour y est sombre, tendre et plein d'une haute estime, le malheur d'autrui éveille dans l'âme du spectateur des sentiments de sympathie (teilnehmende Empfindungen) et fait battre son cœur magnanime pour des mots qui lui sont étrangers. Il est doucement ému et sent la dignité de sa propre nature (Er wird sanft gerührt und fühlt die Würde seiner eigenen Natur)" (E. Kant, Observations sur le beau et sublime, in Euvres philosophiques, I, p. 457) Ce passage de l'émotion à la dignité est précisément ce que conteste Kant en 1790. 
sacrifice qui exemplifieraient le sublime comme le font ailleurs, pour la nature et pour l'art, l'océan déchaîné ou l'immensité des pyramides. Cet effacement de la matière théâtrale est évidemment troublant: à quoi tient dans ce cas le sublime de la tragédie? Comment devra-t-il (et pourra-t-il) se présenter sur la scène?

Kant répond à cette question dans la «Remarque générale». Juste après avoir évoqué la forme tragique, il précise que le sublime ne peut surgir que si le spectateur a rapport à une «manière de penser (Denkungsart)» ${ }^{42}$ qui s'enracine dans les "Idées morales» et que, du côté de l'objet cette fois, s'impose un «mode de présentation (Darstellungsart) totalement négatif vis-à-vis du sensible ${ }^{43}$. Ce dernier point est capital: la naissance du sentiment sublime ne saurait dépendre de la "présentation positive» d'une situation humaine émouvante ou fascinante, qui fixerait le spectateur sur une vision spectaculaire et agiterait en lui des émotions. Le sentiment sublime consiste au contraire à dépasser l'image pour prendre conscience de sa propre dimension morale: «une simple présentation négative, mais qui cependant élargit l'âme", écrit Kant ${ }^{44}$. La référence alors mobilisée est parlante, d'abord parce qu'il s'agit d'un texte, et ensuite parce qu'il est le texte sublime par excellence, à savoir le premier commandement de la Bible, "Tu ne feras point d'image», qui est aussi un principe transcendantal de la poétique du sublime: l'absence d'image, ou du moins l'absence de toute image qui, fixant la pensée, ferait obstacle au retour vers le soi moral - comme si l'identification avec le héros tragique, le Mitleiden, devait toujours être supplanté par la conscience de soi comme porteur des Idées et de l'impératif catégorique. C'est la capacité de la scène à ne pas faire image où à laisser l'image présenter en soi quelque chose d'inimaginable, une «dimension d'illimité» en sus de la beauté des formes, qui activera la dynamique spéculaire nécessaire à ce que le $\$ 52$, du fait de cette négativité, appelle "présentation du sublime» ${ }^{45}$.

42. E. Kant, Critique de la faculté de juger, p. 257. C'est Kant qui souligne.

43. Ibid. Le $\$ 29$ a rattaché le sublime aux «Idées éthiques» et aux «Idées (pratiques)» (p. 248).

44. Ibid., p. 258.

45. Le sublime induit en effet une crise de la (re)présentation. L'idée de "présentation du sublime» est à mettre en relation avec l'argument de la "Remarque générale» selon lequel les Idées elles-mêmes ne sont pas présentables, cette impossibilité de présenter l'Idée étant justement ce qui se présente dans le sublime (Ibid., p. 251). Présenter 


\section{La tragédie et l'Aufklärer}

La situation est claire désormais: les conceptions psychologiques de la tragédie en manquent la sublimité parce qu'elles en méconnaissent le lien à la raison pure pratique. Lactivation de ce lien suppose deux conditions, subjective et objective, que nous venons d'exposer: un spectateur éveillé moralement et une image qui renvoie à l'au-delà de l'image. Les $\$ \$ 29$ et 52 s'accordent explicitement sur la condition subjective, à savoir la présence des «Idées morales» à l'esprit du spectateur. Mais le $\$ 52$ précise aussi comment la condition objective peut être satisfaite en la replaçant dans un devenir où beauté et sublimité ne sont plus simplement associées, mais surgissent et s'engendrent dans le temps de l'Histoire.

Kant, c'était notre point de départ, posait la question de savoir si la liaison du beau et du sublime pouvait donner lieu à une œuvre à la fois belle et unifiée. Quelle est sa réponse? Il assigne à la forme de l'œuvre d'art une double tâche: elle doit assurer l'unité finale de l'œuvre, c'est-àdire ramener la diversité de ses parties à l'unité d'une signification; mais, par là, elle est aussi ce qui produit du côté du sujet percevant un plaisir qui aura un effet sur ce dernier et le préparera à faire, à terme, l'expérience simultanée du beau et du sublime. L'effet esthétique n'est plus ici simplement ponctuel, mais il engage un devenir. C'est ce que veut dire Kant, lorsqu'il écrit que «le plaisir est en même temps culture et dispose (stimmt) l'esprit à des Idées en le rendant par conséquent capable d'éprouver (empfänglich macht) bien davantage de plaisirs et de divertissements de ce type». Cette remarque signifie que la contemplation $\mathrm{du}$ beau nourrit un progrès qui permettra de mettre l'esprit en rapport avec des Idées (Kant ne parle toutefois que de «disposition", nous allons y revenir) et par-là même de développer le goût et d'élargir la palette des plaisirs du spectateur. Cette idée doit être mise en relation avec le $\$ 29$ qui rappelait que la «culture (Kultur)» est nécessaire à l’appréciation du sublime d'un paysage de montagne, en l'occurrence un glacier, sans quoi ce dernier paraîtrait simplement effrayant, comme aux yeux du «brave [et inculte] paysan savoyard», incapable de le considérer

le sublime, c'est donc présenter dans l'image ce qui est plus grand ou plus fort que toute image, l'intimité morale. Sur les interprétations postmodernes de ce passage, voir R. Doran, The Theory of Sublime from Longinus to Kant, p. 270. 
esthétiquement ${ }^{46}$. Or c'est cette situation que l'expérience du beau permet de surmonter, en cultivant et en disposant aux Idées, nécessaires à la saisie de ce plaisir complexe qu'offre la tragédie, à la fois belle et sublime. Le $\$ 52$ reprendrait donc la dialectique dont la «Remarque générale» énonçait le principe lorsqu'elle insistait sur le fait que les sentiments du beau et du sublime, au-delà de leur nécessaire distinction, peuvent se réunir «dans le même sujet en trouvant leur finalité à travers une relation au sentiment moral ${ }^{47}$. Kant distinguait alors entre le beau qui nous initie au désintéressement, qui nous apprend à voir hors de toute perspective théorique ou utilitaire, et le sublime qui va plus loin en nous apprenant à aimer ce qui va "contre notre intérêt» et en nous orientant "vers les fins de la raison pratique» ${ }^{48}$. Les rapports entre beau et culture évoqués dans le $\$ 52$ s’inscrivent dans ce scénario: en nous initiant au désintéressement, l'expérience du beau ne donne pas directement accès aux Idées, mais elle fait place pour elles, écartant ce qui pourrait y faire obstacle - ainsi la peur qui emplit l'âme du paysan savoyard devant le glacier. Une fois cette «disposition" acquise, les Idées pourront se donner au sujet esthétique et lui permettre de s'élever de l'expérience du beau à celle du sublime.

Il y a là pour le théâtre deux conséquences. La première, c'est que la pièce de théâtre aura sa place, au côté de l'éloquence, dans le premier moment de cette histoire esthético-morale, au titre de ces "divertissements» qui nourrissent le cercle vertueux de la culture et du plaisir ${ }^{49}$. La seconde, c'est que la tragédie, si elle doit justement être préparée par cette culture de la beauté des formes qui dispose à la considération des "Idées morales", appartiendra à un moment distinct, historiquement postérieur. Une question simple se pose alors: cette époque est-elle arrivée? Où se situe-t-elle par rapport au moment où Kant écrit sa troisième Critique?

46. E. Kant, Critique de la faculté de juger, p. 247 sq.

47. Ibid., p. 251.

48. Ibid. Voir aussi la "Quatrième proposition» d'une Idée d'une histoire universelle au point de vue cosmopolitique où la formation du goût précède la formation d'une "façon de penser» qui mènera à terme à des "principes pratiques déterminés» (E. Kant, Euvres philosophiques, vol. 2, p. 192).

49. C'est-à-dire le plaisir de se cultiver: voir E. Kant, Anthropologie d'un point de vue pragmatique, $\$ 63$, p. 98. 
Pour comprendre la situation en 1790 , il faut s'attarder sur les lignes qui concluent le premier temps du $\$ 52$. Elles traitent de l'état d'esprit du spectateur dont la perception se bornerait à ce qui est inessentiel dans l'œuvre, c'est-à-dire la «matière de la sensation (l'attrait ou l'émotion)». Le spectateur bourgeois de la "Remarque générale», on s'en souvient, était bien content de s'être désennuyé; il avait joui d'une tragédie réduite à sa dimension matérielle et émotionnelle. Le spectateur introduit dans le $\$ 52$ est quant à lui dans un autre état d'esprit: il désespère de ce qu’il voit. Kant écrit :

La jouissance [...] n'apporte rien à l'Idée, émousse l'esprit, fait peu à peu éprouver du dégoût envers son objet et rend l'âme insatisfaite d'elle-même et chagrine par la conscience de sa disposition qui, selon le jugement de la raison, apparaît contraire à toute finalité.

Le dégoût dont il est question ici est celui d'un sujet qui a pris conscience des Idées et attend un spectacle à sa mesure, mais qui se trouve insatisfait. Insatisfaction qui n'est pas une simple absence de plaisir, mais une «insatisfaction de soi", un rabaissement, l'exact contraire de "l'estime de soi " ${ }^{50}$ qu'est censé faire naître le sublime et qu'en droit le spectacle devrait lui fournir. À l'évidence, le spectateur du $\$ 52$ est l'Aufklärer qui, sous l'effet d'un développement historique de la culture esthétique, est en contact étroit avec les «Idées morales» et ne peut plus s'en tenir aux jouissances pathologiques. S'il désespère, c'est parce que le spectacle qu'on lui offre n'est pas relié à ces Idées, parce qu'il arrive, stipule Kant à la ligne suivante, que "les beaux-arts ne [soient] pas, de près ou de loin, associés à des Idées morales» et se mettent en contradiction avec la destination de l'être raisonnable. Le problème n'est donc pas du côté du spectateur; il est du côté des arts dont le "destin", souligne le $\$ 52$, est alors de déchoir en précipitant l'homme des Lumières avec lui. Pourquoi? Parce que si le théâtre amoral ou pathologique n'engendre que de la «jouissance» et si celle-ci engendre l'insatisfaction de soi chez l'Aufklärer, cette insatisfaction de soi favorisera à son tour la recherche de la "distraction" à titre de consolation. Pour surmonter la déception d'un spectacle qui n'est pas à sa mesure, le spectateur frustré s'abrutira de satisfactions superficielles, s'éloignant de cette destination morale dont il avait pourtant conscience. En ce sens, le spectateur du $\$ 29$ est l'état

5o. Voir E. Kant, Critique de la faculté de juger, \$28, p. 244. 
final - c’est-à-dire délabré - du spectateur du \$52. Il y a là un déphasage historique: l'expérience du beau, prise dans un devenir culturel, permet au sujet de se mettre en rapport avec les Idées; l'esthétique contribue à révéler à lui-même le sujet moral qui, en retour, pose son exigence; il demande aux arts ce qui est au-delà de la matière, mais aussi de la forme belle - au-delà de l'image -, en accord avec sa destination ultime; or les arts, sans lien aux «Idées morales», sont défaillants et exposent le sujet au déclin...

Que veut nous dire Kant sur la tragédie? Qu'elle constitue bien une tentative pour donner satisfaction à l'idée primitive de l'art qui est d'ouvrir à la morale, en nous hissant jusqu'à l'expérience du sublime; mais que pareille tragédie ne sera effective qu'à la condition d'un lien intime avec les Idées qu'a certes développé le spectateur des Lumières, mais dont est manifestement dépourvu l'art théâtral de la fin du XVIII ${ }^{e}$ siècle ${ }^{51}$. La tragédie devrait être le sommet de l'histoire de l'art et de l'expérience esthétique comme expression de la culture humaine; malheureusement, du fait de la limitation pathologique qui caractérise son expérience actuelle, elle est le lieu de sa réduction et de sa dévalorisation. À cet égard, la phrase conclusive du $\$ 52$ pourrait s'entendre comme un aveu d'échec et une invitation à se détourner des arts:

D’une façon générale, ce sont les beautés de la nature qui s'accordent le plus avec l'intention première de l'art, quand on est dès l'enfance accoutumé à les observer, à les apprécier et à les admirer.

Kant se replierait sur les satisfactions naturelles dont l'atteinte n'est pas conditionnée par une histoire esthético-morale et n'exige rien d'autre qu'une habitude contractée dès le plus jeune âge. Il reviendrait à une voie plus directe: le plaisir pris au beau naturel est, selon le $\$ 42$, le révélateur de la disposition morale de l'homme ${ }^{52}$, quand l'art ne peut espérer atteindre ce but, on l'a vu, qu'au terme d'une dialectique périlleuse.

Mais il est encore une autre façon d'apprécier la conception kantienne du théâtre proposée dans la troisième Critique: le théâtre sous sa forme

5I. Le «de près ou de loin» qui ouvre le dernier temps du $\$ 52$ semble indiquer que tous les beaux-arts ou toutes les œuvres d'art, quand elles sont reliées aux Idées morales, ne le sont pas de la même façon et qu'une disparité s'introduit ici.

52. Ibid., \$42, p. 284 sq. Rappelons que le $\$ 42$ est aussi celui qui introduit à la réflexion sur l'art et les beaux-arts qui s'étendra des $\$ \$ 43$ à 54 . 
tragique s'y trouve du côté du fait et du droit, du sensible et de l'Idée, de l'histoire et du suprahistorique. Menacé de voir son effet se réduire à une catharsis pathologique, la tragédie est vidée par Kant de ses images et de sa logique d'identification pour être convertie en un idéal régulateur dont il paraît bien difficile d'envisager la concrétisation empirique. En nous invitant à évaluer la conception de la tragédie dominante à son époque sur la base d'une exigence esthétique et morale, Kant nous met en rapport avec ce qu'il faudrait appeler une «Idée» de la tragédie, caractérisée par la conscience sublime de la liberté humaine; de ce point de vue, il annonce Schelling qui émancipera pleinement le concept du tragique de l'approche poéticienne de la tragédie ${ }^{53}$. Fine pointe de la théorie des arts de la Critique de la faculté de juger, le $\$ 52$ constituerait un tournant où s'articule une réflexion sur la forme théâtrale de la tragédie, sur son inscription dans le présent de l'Aufklärung et sur le tragique comme expérience de l'homme ${ }^{54}$.

Olivier Dubouclez

Université de Liège

53. Voir P. Szondi, Essai sur le tragique, p. 9.

54. Pour une perspective plus générale sur la forme tragique et le problème de sa réactualisation dans l'histoire, voir D. Bouvier, «Mémoire de la cité et mémoire de la tragédie». 


\section{BIBLIOGRAPHIE}

\section{Textes}

Kant, Immanuel, Kant's gesammelte Schriften, hrsg. v. der Königlich Preussischen Akademie der Wissenschaften, 23 Bd., Berlin/ Leipzig, Georg Reimer/Walter de Gruyter, 1902-1955.

-, Anthropologie d'un point de vue pragmatique, trad. Michel Foucault, Paris, Vrin, 1964.

-, Euvres philosophiques, sous la direction de Ferdinand Alquié, 3 vols, Paris, Gallimard, 1980-1986 (Bibliothèque de la Pléiade).

-, Critique de la faculté de juger, trad. et intr. Alain Renaut, Paris, Garnier Flammarion, 2000.

Rousseau, Jean-Jacques, Lettre à d'Alembert sur les spectacles, in CEuvres complètes, V, Paris, Gallimard, 1995 (Bibliothèque de la Pléiade).

\section{Travaux}

BÉGot, Jacques-Olivier, «La naissance du tragique, enfanté par l'esprit du sublime", Revue germanique internationale, 18 (2013), p. 75-90.

Bouvier, David, "Mémoire de la cité et mémoire de la tragédie», in Michael Groneberg (éd.), Penser la scène, Études de Lettres (306) 2018, p. 81-98.

Château, Jean-Yves, Pourquoi un septième Art? Cinéma et philosophie, Paris, Puf, 2008.

De Saint Girons, Baldine, «Du sublime comme assomption du tragique: Longin contre Aristote", in Id., Le sublime de l'Antiquité à nos jours, Paris, Desjonquères, 2005, p. 36-49.

Doran, Richard, The Theory of Sublime from Longinus to Kant, Cambridge, Cambridge University Press, 2015. 
Dumouchel, Daniel, Kant et la genèse de la subjectivité esthétique, Paris, Vrin, 1999.

Gemeaux, Christine de, De Kant à Adam Müller (1790-1815): éloquence, espace public et médiation, Paris, Presses universitaires de la Sorbonne, 2012.

Groneberg, Michael, «Le Gesamtkunstwerk et l'homme entier. Essai sur l'actualité de l'art total», in Michael Groneberg (éd.), Penser la scène, Etudes de Lettres, (306) 2018, p. 27-46.

Kirknopelto, Esa, Le théâtre de l'expérience. Contributions à la théorie de la scène, Paris, Presses universitaires de la Sorbonne, 2008.

Kuenn, Manfred, Kant. A Biography, Cambridge, Cambridge University Press, 2001.

MARIE, Laurence, Inventer l'acteur. Émotions et spectacle dans l'Europe des Lumières, Paris, Pressses universitaires de la Sorbonne, 2018.

NANCY, Jean-Luc, "L'offrande sublime», in Jean-François Courtine et al. (dir.), Du sublime, Paris, Belin, 2009, p. 37-75.

Stroud, Scott R., Kant and the Promise of Rhetoric, Pennsylvania State University Press, University Park, 2014.

Szondi, Peter, Essai sur le tragique, trad. J.-L. Besson, M. Gondicas, P. Judet de La Combe et J. Jourdheuil, Belval, Circé, 2003. 